\title{
Michael, Hendrik: Die Sozialreportage als Genre der Massenpresse. Erzählen im Journalismus und die Vermittlung städtischer Armut in Deutschland und den USA (1880-1910).
}

\author{
Bremen: edition lumière 2020. 536 Seiten. Preis: $€$ 44,80
}

\section{Walter Hömberg}

Angenommen: 6. März 2021 / Online publiziert: 23. März 2021

(C) Der/die Autor(en) 2021, korrigierte Publikation 2021

Die Reportage ist „das Kronjuwel des Journalismus“. Diese Formulierung stammt von Georg Brunold, dem wir ein bibliophiles Meisterwerk verdanken. Sein voluminöses Buch Nichts als die Welt versammelt ,Reportagen und Augenzeugenberichte aus 2500 Jahren“ (Berlin 2009). Der Herausgeber spannt den Bogen von Herodot, Thukydides, Hippokrates, Xenophon und Platon bis zu Sophia Woodman, David Kermani, Hans Magnus Enzensberger, Karl-Markus Gauss und Andreas Langenbacher. Wenn die Reportage als ,wahrhaft universelle literarische Gattung“ gesehen wird, haben solche Zeitreisen ihren eigenen Reiz.

Allerdings: Als journalistische Form ist die Reportage erst im 19. Jahrhundert massenwirksam geworden. Im Übergang vom schriftstellerischen zum redaktionellen Journalismus haben sich nicht nur die berufssoziologischen Rahmenbedingungen, sondern auch die Berichterstattungsmuster verändert. Eine Nähe zur Form der Reportage kann man in den Reiseerzählungen beobachten, die in großer Zahl zur Zeit des Vormärz entstanden sind. Die Schriftsteller im Umkreis des Jungen Deutschland nutzten die verbesserten Verkehrsverhältnisse, um der Enge der deutschen Fürstenparzellen zu entfliehen. Kritik an heimischen Missständen, die die Zensur verhindern wollte, wurde gern indirekt durch Berichte über fremde Länder geäußert. Reiseskizzen waren nicht nur ein beliebtes Transportmittel für Information und Unterhaltung, sondern dienten auch dem Ideenschmuggel.

Hendrik Michael zeigt sich in seiner Studie skeptisch gegenüber Versuchen, die Ahnengalerie der Reportage bis in die Antike zurückzuverfolgen. Er sieht ihren Ursprung aus gutem Grund in den politischen und sozialen Umbruchprozessen des 19. Jahrhunderts. Industrialisierung, Urbanisierung und Technisierung - auf diese

Prof. em. Dr. W. Hömberg $(\bowtie)$

Lehrstuhl für Journalistik I, Katholische Universität Eichstätt-Ingolstadt,

Ostenstraße 25, 85071 Eichstätt, Deutschland

E-Mail: walter.hoemberg@ku.de 
Megatrends reagierten auch die damals verfügbaren Massenmedien. Die Großstadtpresse erweiterte ihr inhaltliches Angebot um lokale Berichterstattung und nahm neue Zielgruppen ins Visier. Das galt sowohl für die US-amerikanische als auch mit zeitlicher Verzögerung - für die deutsche Massenpresse.

Michael will in seiner Studie ,untersuchen, welche Leistung die Sozialreportage als Genre der Massenpresse erfüllte, um journalistischen Medien Umsetzungsstrategien zu bieten, sich mit dem Thema Armut auseinanderzusetzen und diesen Sachverhalt einer massenmedialen Öffentlichkeit zu vermitteln“ (S. 17). Die Gliederung der Arbeit, die als Dissertation an der Universität Bamberg entstanden ist, folgt dem klassischen Muster. Am Beginn steht ein Forschungsüberblick. Der Verfasser zeigt sich dabei gut informiert über die einschlägigen Publikationen zur Massenpresse und zur Reportage im deutschen und angloamerikanischen Sprachraum.

Sehr detailliert erläutert Michael sein Vorgehen. Das Kapitel „Quellenerhebung, Methodik, analytische Grundlegung“ umfasst mehr als 70 Druckseiten. Langer Rede kurzes Resultat: Mit Hilfe einer qualitativen Inhaltsanalyse wird eine Auswahl von insgesamt 427 Artikeln im Hinblick auf Berichterstattungsmuster, diskursive Strategien und journalistische Funktionen untersucht. Der inhaltliche Fokus liegt auf Reportagen zum Themenfeld städtische Armut. Das Untersuchungsmaterial stammt aus je sechs auflagenstarken amerikanischen und deutschen Großstadtzeitungen, die zwischen 1880 und 1910 in New York und Berlin erschienen sind, sowie aus einigen Magazinen und Reportagesammlungen in Buchform aus dem gleichen Zeitraum. Das differenzierte Analysemodell verbindet Kategorien textlinguistischer Erzählforschung mit solchen aus der kommunikations- und medienwissenschaftlichen Berufsforschung.

Bei der Auswertung wird unterschieden zwischen Binnenstruktur (Darstellungsformen und Herstellung von Authentizität) und Außenstruktur (Realisierung im Kontext von Produktion und Rezeption). Die Darstellung der Ergebnisse legt den Schwerpunkt auf die Reihe „True Stories of the News“ der Zeitung The World (75 Beiträge) und die Serie „Aus dem Dunklen Berlin“ in der Berliner Morgenpost (48 Beiträge). Der Verfasser erläutert im (streckenweise ermüdenden) Detail die unterschiedlicher Erzählstrategien. Eine solche Vorgehensweise ist aus vielen Inhaltsanalysen bekannt. Innovativ hingegen, dass unter der Rubrik „Außenstruktur“ die journalistischen Produktionsroutinen und die Rahmenbedingungen der Nutzung thematisiert werden. Redaktionelle Strukturen, Arbeitsweisen und Ressourcen geraten ebenso ins Blickfeld wie Vertriebsformen und Rezeptionsmuster. Zusammenfassend versucht Michael, die Armutsdarstellung im Genre Sozialreportage als Schnittmenge der drei Berichterstattungsmuster Sensationsjournalismus, Investigativjournalismus und Literarischer Journalismus analytisch zu verorten.

Ein wichtiges Kriterium für die Glaubwürdigkeit der Sozialreportagen ist die Authentizität der Darstellung. Der Verfasser erläutert eingehend die unterschiedlichen Modi des Realitätsbezugs. Er muss einräumen, dass der Wahrheitsgehalt der Reportagen im Einzelfall nicht überprüfbar ist - es bleibt lediglich, a feeling of truth".

Wie sehr dies täuschen kann, hat hierzulande der Fall des Spiegel-Redakteurs Claas Relotius gezeigt. Michael Haller, auf den sich Hendrik Michael an manchen Stellen beruft, hat sich in der kürzlich erschienen 7. Auflage seines komplett 
überarbeiteten Lehrbuchs Die Reportage (Köln 2020) eingehend befasst mit „den Spielräumen zwischen dem Faktualen und dem Fiktionalen, zwischen der subjektiven Sicht und der Verdichtung des Stoffes im Text". Er erläutert dabei seine in früheren Auflagen vertretenen Positionen und modifiziert sie teilweise.

Während die Erfindungen von Relotius zunächst durch einen kritischen Kollegen und später dann durch eine von der Redaktion eingesetzte Aufklärungskommission bloßgelegt wurden, kann ein Medienhistoriker retrospektiv nur selten Fakes und Fiktionen aufdecken. Bei der Reporterikone Egon Erwin Kisch ist dies in einigen Fällen gelungen. Wenn die Glaubwürdigkeit eines Autors oder eines Beitrags jedoch nicht durch eklatante Widersprüche oder überzeugende Gegendarstellungen unterminiert ist, bleibt nur die Wahrheitsvermutung. Dass ein solcher Vertrauensvorschuss selbst von sogenannten Qualitätsmedien missbraucht werden kann, haben indes mannigfache Beispiele in den letzten Jahrzehnten gezeigt.

Das vorliegende Buch greift viele Impulse von Hannes Haas auf, dessen innovative Habilitationsschrift über die Erkundung sozialer Wirklichkeit Maßstäbe gesetzt hat (Empirischer Journalismus, Wien 1999; siehe meine Rezension in Publizistik, 2/2000). Michaels Studie, die im vergangenen Jahr mit dem Nachwuchsförderpreis der DPuK-Fachgruppe Kommunikationsgeschichte ausgezeichnet wurde, überzeugt insgesamt sowohl durch ihre methodische Stringenz als auch durch ihren kulturvergleichenden Ansatz. Der Autor hat eine Fülle von Literatur verarbeitet (das Quellenund Literaturverzeichnis umfasst 49 Seiten); auch die enorme Zahl der Fußnoten zeugt davon (durchschnittlich 5 pro Textseite, insgesamt 2291).

Allerdings: Eine „Handlungsanleitung für die publizistisch interessierten Verleger unserer Tage“, die im Vorwort von Rudolf Stöber versprochen wird, liefert dieses Buch nicht. Und auch die dort herbeigewünschten Leser ,unter heutigen Verlegern und Publizisten“ dürfte es wohl kaum finden. Dazu ist es zu sehr in einer hermetischen Wissenschaftsdiktion verfasst.

Funding Open Access funding enabled and organized by Projekt DEAL.

Open Access Dieser Artikel wird unter der Creative Commons Namensnennung 4.0 International Lizenz veröffentlicht, welche die Nutzung, Vervielfältigung, Bearbeitung, Verbreitung und Wiedergabe in jeglichem Medium und Format erlaubt, sofern Sie den/die ursprünglichen Autor(en) und die Quelle ordnungsgemäß nennen, einen Link zur Creative Commons Lizenz beifügen und angeben, ob Änderungen vorgenommen wurden.

Die in diesem Artikel enthaltenen Bilder und sonstiges Drittmaterial unterliegen ebenfalls der genannten Creative Commons Lizenz, sofern sich aus der Abbildungslegende nichts anderes ergibt. Sofern das betreffende Material nicht unter der genannten Creative Commons Lizenz steht und die betreffende Handlung nicht nach gesetzlichen Vorschriften erlaubt ist, ist für die oben aufgeführten Weiterverwendungen des Materials die Einwilligung des jeweiligen Rechteinhabers einzuholen.

Weitere Details zur Lizenz entnehmen Sie bitte der Lizenzinformation auf http://creativecommons.org/ licenses/by/4.0/deed.de.

Prof. em. Dr. W. Hömberg war Lehrstuhlinhaber für Journalistik und Kommunikationswissenschaft an den Universitäten Bamberg und Eichstätt und hat lange Zeit als Gastprofessor an der Universität Wien gelehrt. 